\title{
Desabastecimiento de antidiabéticos y antihipertensivos en el contexto de la etapa inicial de la pandemia por la COVID.19 en Perú
}

\section{Shortage of antidiabetic and antihypertensive in the context of the initial stage of the COVID-19 pandemic in Peru}

DOI

https://doi.org/10.35434/rcmhnaaa.2021.14Sup1.1152

\section{RESUMEN}

Introducción: Un adecuado abastecimiento de medicamentos en los establecimientos de salud aumentará la posibilidad de un adecuado control de la hipertensión y la diabetes. Objetivo: Determinar el desabastecimiento de antidiabéticos y antihipertensivos a nivel nacional en el contexto de la etapa inicial de la pandemia por la COVID19 en Perú. Material y métodos: Análisis del "Sistema integrado de suministro de medicamentos e insumos médicos quirúrgicos" (SISMED) entre el 13 de junio y 15 de julio del 2020, de acuerdo a la lista del "Petitorio nacional único de medicamentos de medicamentos esenciales” (PNUME) del Ministerio de Salud. Resultados: Entre el $16 \%$ y $24 \%$ de las regiones están totalmente desabastecidos en al menos un antidiabético y entre 4 y $96 \%$ en al menos un antihipertensivo. El antidiabético más desabastecido fue la Metformina de $500 \mathrm{mg}$ y los antihipertensivos más desabastecidos fueron Labetalol $5 \mathrm{mg} / \mathrm{ml}$ iny, Atenolol $50 \mathrm{mg}$ tab y Carvedilol 6,5 mg tab. El desabastecimiento fue porcentualmente mayor en hospitales y los institutos especializados en comparación con centros y puestos de salud según región geográfica. Conclusiones: Existe un desabastecimiento de antihipertensivos y antidiabéticos en los establecimientos de salud, el cual es heterogéneo en las diferentes regiones del Perú.

Palabras Clave: Antihipertensivos; Antidiabéticos; Accesibilidad a los Servicios de Salud; establecimientos de salud (Fuente: DeCS-BIREME).

\section{ABSTRACT}

Background: An adequate supply of medicines in health establishments will increase the possibility of adequate control of hypertension and diabetes. Objective: To determine the shortage of antidiabetic and antihypertensive drugs at the national level in the context of the initial stage of the COVID-19 pandemic in Peru. Material y methods: Analysis of the "Sistema Integrado de Suministro de Medicamentos e Insumos Médicos Quirúrgicos" (SISMED) Database, between June 13th and July 15th, 2020, according to the "National list for medicines of essential medicines" (PNUME) of Ministry of health. Results: and between 4 and $96 \%$ of the departments have a total shortage of at least one antidiabetic, and $4 \%$ and $96 \%$ of at least one antihypertensive. The most depleted antidiabetic was Metformin 500 $\mathrm{mg}$, and the most depleted antihypertensive drugs were Labetalol $5 \mathrm{mg} /$ $\mathrm{ml}$ iny, Atenolol $50 \mathrm{mg}$ tab and Carvedilol $6.25 \mathrm{mg}$ tab. The percentage of distribution was higher in hospitals and specialized institutes in comparison with primary health facilities. Conclusions: There is a shortage of antihypertensive and antidiabetic drugs in health centers in Peru.

Keywords: Antihypertensive Agents; Hypoglycemic Agents; Health Services Accessibility; Health Facilities. (Source: DeCS-BIREME).

Percy Herrera-Añazco ${ }^{1,2, a}$, Germán Valenzuela-Rodríguez ${ }^{3.4, a}$, Luciana Torres-Pesantes ${ }^{5, a}$, Carlos J. Toro-Huamanchumo

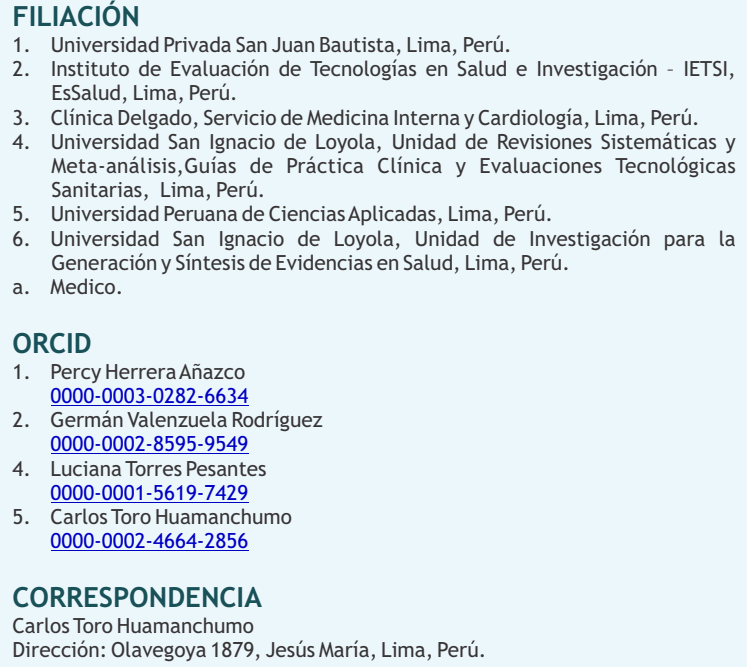

1. Universidad Privada San Juan Bautista, Lima, Perú.

2. Instituto de Evaluación de Tecnologías en Salud e Investigación - IETSI, EsSalud, Lima, Perú.

3. Clínica Delgado, Servicio de Medicina Interna y Cardiología, Lima, Perú. 4. Universidad San Ignacio de Loyola, Unidad de Revisiones Sistemáticas y Meta-análisis,Guías de Práctica Clínica y Evaluaciones Tecnológicas Sanitarias, Lima, Perú.

5. Universidad Peruana de Ciencias Aplicadas, Lima, Perú.

6. Universidad San Ignacio de Loyola, Unidad de Investigación para la Generación y Síntesis de Evidencias en Salud, Lima, Perú.

a. Medico.

ORCID

1. Percy Herrera Añazco 0000-0003-0282-6634

2. Germán Valenzuela Rodríguez 0000-0002-8595-9549

4. Luciana Torres Pesantes 0000-0001-5619-7429

Carlos Toro Huamanchumo

0000-0002-4664-2856

CORRESPONDENCIA

Carlos Toro Huamanchumo

Dirección: Olavegoya 1879, Jesús María, Lima, Perú.

EMAIL

silamud@gmail.com

CONFLICTOS DE INTERÉS

Los autores niegan conflictos de interés.

CONTRIBUCIÓN DE LOS AUTORES

PHA, CTH, GVR y LTP han participado en la concepción del artículo, la recolección de datos, su redacción y aprobación de la versión final. Además, CTH y GVR realizaron el análisis de datos.

\section{FINANCIAMIENTO}

Autofinanciamiento.

\section{REVISIÓN DE PARES}

Recibido: 19/05/2021

Aceptado: 02/08/202

\section{COMO CITAR}

Herrera-Añazco, P., Valenzuela-Rodríguez, G., Torres-Pesantes, L., \& ToroHuamanchumo, C. Desabastecimiento de antidiabéticos y antihipertensivos en el contexto de la etapa inicial de la pandemia por la COVID-19 en Perú. Revista Del Cuerpo Médico Hospital Nacional Almanzor Aguinaga Asenjo, 2021, 14(Sup1), 22 - 27. https://doi.org/10.35434/rcmhnaaa.2021.14Sup1.1152 


\section{INTRODUCCIÓN}

La hipertensión arterial (HTA) y la diabetes mellitus (DM) son dos enfermedades no comunicables consideradas como problemas de salud pública a nivel mundial por su tendencia creciente y por los costos que su manejo implica para los sistemas de salud de los países, sobre todo en los de ingresos medios y bajos ${ }^{(1-3)}$.

En Perú, la prevalencia de la HTA en pacientes que acuden a establecimientos de salud del Ministerio de Salud (MINSA) aumentó en los últimos años, sin embargo, disminuyó el autoconocimiento y el control de esta enfermedad ${ }^{(4,5)}$. La prevalencia de DM también aumentó(6), que en el caso de pacientes menores de 30 años, significó un aumento de diez veces desde el 2005 al $2018^{(7)}$. A pesar de que la prevalencia de DM es menor que en otros países de Latinoamérica ${ }^{(8)}$, hay evidencia de un pobre control metabólico en los pacientes y de un inadecuado despistaje de las complicaciones tardías de la enfermedad en nuestro país ${ }^{(9)}$. Los problemas de control de la enfermedad y manejo de complicaciones tanto para HTA y DM son multifactoriales, dentro de los que se encuentran la desarticulación de las estrategias sanitarias del MINSA al detectar nuevos casos o a un desabastecimiento de medicamentos, como consecuencias de nuestro fragmentado sistema de salud ${ }^{(10)}$

El sistema de salud peruano presenta múltiples problemas estructurales y administrativos que limitan la capacidad resolutiva de los establecimientos de salud del MINSA ${ }^{(10)}$, y que son los que enfrentan la actual crisis sanitaria por el COVID-19. A pesar de que Perú fue uno de los primeros países en el continente en establecer medidas de confinamiento social ${ }^{(11)}$, los resultados son limitados, siendo a la fecha el país con mayor exceso de muertes en el mundo ${ }^{(12)}$. Aunque las cifras oficiales actualmente superan las 200000 muertes $^{(13)}$, se sospecha que hay un subregistro y con la posibilidad de que haya una sustancial cantidad de fallecimientos por otras enfermedades no tratadas, pues los establecimientos de salud priorizaron la atención de pacientes con infección por COVID-19 ${ }^{(12)}$. Esta falta de tratamiento no solo está limitada a la consulta médica sino al desabastecimiento de medicamentos para el manejo de enfermedades, tales como la HTA y la DM, que ya eran un problema antes de la crisis sanitaria actual ${ }^{(14,15)}$. No obstante, a la fecha, este problema no se ha cuantificado. Por lo que el objetivo del presente estudio es determinar el desabastecimiento de los antidiabéticos y antihipertensivos según nivel de establecimiento de salud en el Ministerio de Salud en el contexto de la primera etapa de la pandemia por la COVID-19.

\section{MATERIALYMÉTODOS}

Estudio transversal de la disponibilidad de antidiabéticos y antihipertensivos en los establecimientos de salud a nivel nacional entre el 15 de junio y el 15 de julio del 2020, etapa en la que finalizó la cuarentena total impuesta por el gobierno central a raíz de la crisis por el COVID-19. Se excluyeron los antidiabéticos, antihipertensivos y la combinación de medicamentos que no se encontraron en el "Petitorio nacional único de medicamentos esenciales para el sector salud" (PNUME). De igual forma, se excluyó a la Desmopresina, que, si bien está en el petitorio como antidiabético, es usada para el tratamiento de la Diabetes
Insípida y no para Diabetes Mellitus, que es el objetivo del estudio.

Los antidiabéticos y antihipertensivos fueron obtenidos del PNUME

(https://repositorio.digemid.minsa.gob.pe/bitstream/hand le/DIGEMID/149/PNUME_2018_Ed.pdf?sequence=3\&isAllow ed=y) El PNUME es el documento normativo oficial del MINSA referido a la prescripción, dispensación y utilización de medicamentos en los diferentes niveles de atención de los establecimientos de salud de Perú, y considera antidiabéticos y antihipertensivos esenciales que deben estar en los establecimientos de salud a nivel nacional y que se muestran en la tabla 1.

La disponibilidad de medicamentos fue obtenida de los datos del Sistema Integrado de Suministros de Medicamentos e Insumos Médicos-Quirúrgicos (SISMED): https://appsalud.minsa.gob.pe/consolida/portalsismed/Re pPrecioMedicamento.aspx. El SISMED clasifica los medicamentos de acuerdo al Sistema de Clasificación Anatómica Terapéutica Química de la Organización Mundial de la Salud (16) y recopila informes mensuales de inventarios de medicamentos de los establecimientos de salud públicos. EI SISMED remiten su información a la Dirección General de Medicamentos Insumos y Drogas (DIGEMID) del MINSA. Estos informes son susceptibles a auditorías administrativas, lo que obliga a las instituciones a registrar la información de una manera precisa y contrastable y son, la fuente oficial de información sobre el abastecimiento de medicamentos del MINSA, utilizados para la definición de indicadores, para los procesos de monitoreo y evaluación, así como los procesos de adquisición y distribución de medicamentos desde el año 2001.

Analizamos la disponibilidad de los antidiabéticos y antihipertensivos en todos los establecimientos de salud del país distribuidos en las 25 regiones políticas de Perú: 24 departamentos y una provincia constitucional, así como sus tres regiones geográficas: costa, sierra y selva. El desabastecimiento para cada medicamento por establecimiento se definió bajo dos formas: 1) cuando no apareció el establecimiento en la búsqueda en el SISMED, o 2) apareció el establecimiento, pero reportó nulidad de stock.

La información obtenida fue sintetizada en cuadros de doble entrada, considerando los medicamentos, tipo de establecimiento de salud, regiones políticas y geográficas.

Para evaluar el porcentaje de regiones desabastecidas se dividió el número de departamentos desabastecidos de acuerdo al medicamento sobre 0,25 (24 departamentos + una provincia constitucional) para todos los medicamentos.

Para evaluar la distribución de los medicamentos según región geográfica, se calculó el porcentaje de establecimiento con stock disponible. Para ello, se usó la siguiente fórmula:

\#establecimientos - \#establecimientos sin stock \#establecimientos

Este estudio realizó un análisis de datos secundarios que fueron obtenidos mediante una solicitud a una web de acceso público e informes publicados, por lo que no fue necesario la aprobación de un comité de ética. 


\section{RESULTADOS}

Tabla 1. Medicamentos antihipertensivos y antidiabéticos incluidos en el Petitorio Nacional Único de Medicamentos Esenciales.

\begin{tabular}{|c|c|c|}
\hline Medicamento & Concentración & $\begin{array}{c}\text { Forma } \\
\text { farmacéutica }\end{array}$ \\
\hline \multicolumn{3}{|l|}{ Antihipertensivos } \\
\hline Atenolol & $50 \mathrm{mg}$ & Tableta \\
\hline Carvedilol & $25 \mathrm{mg}$ & Tableta \\
\hline Carvedilol & $6.25 \mathrm{mg}$ & Tableta \\
\hline Carvedilol & $12.5 \mathrm{mg}$ & Tableta \\
\hline Captopril & $25 \mathrm{mg}$ & Tableta \\
\hline Enalapril maleato & $10 \mathrm{mg}$ & Tableta \\
\hline Enalapril maleato & $20 \mathrm{mg}$ & Tableta \\
\hline Labetalol & $5 \mathrm{mg} / \mathrm{ml}$ & Inyectable \\
\hline Hidroclorotiazida & $25 \mathrm{mg}$ & Tableta \\
\hline Metildopa & $250 \mathrm{mg}$ & Tableta \\
\hline Nitroprusiato sódico & $50 \mathrm{mg}$ & Inyectable \\
\hline Losartán potásico & $50 \mathrm{mg}$ & Tableta \\
\hline Amlodipino (como besilato) & $10 \mathrm{mg}$ & Tableta \\
\hline Amlodipino (como besilato) & $5 \mathrm{mg}$ & Tableta \\
\hline Bisoprolol fumarato & $5 \mathrm{mg}$ & Tableta \\
\hline Nifedipino & $30 \mathrm{mg}$ & Tableta \\
\hline Atenolol & $100 \mathrm{mg}$ & Tableta \\
\hline \multicolumn{3}{|l|}{ Antidiabéticos } \\
\hline Glibenclamida & $5 \mathrm{mg}$ & Tableta \\
\hline Insulina humana & $100 \mathrm{UI} / \mathrm{ml}$ & Inyectable \\
\hline Insulina isófona humana (NPH) & $1000 \mathrm{UI} / \mathrm{ml}$ & Inyectable \\
\hline Metformina clorhidrato & $850 \mathrm{mg}$ & Tableta \\
\hline Metformina clorhidrato & $500 \mathrm{mg}$ & Tableta \\
\hline
\end{tabular}

Tabla 2. Desabastecimiento de antidiabéticos según región política.

$\begin{array}{cccc}\text { Región } & \begin{array}{c}\text { Insulina humana (ADN } \\ \text { recombinante) } 100\end{array} & \begin{array}{c}\text { Insulina isófana humana } \\ \text { (NPH) ADN recombinante }\end{array} & \begin{array}{c}\text { Metformina } \\ \text { clorhidrato } 500\end{array} \\ & \mathrm{UI} \text { ml iny } & 100 \mathrm{Ul} \text { iny } & \text { mg tab }\end{array}$

\begin{tabular}{|c|c|c|c|}
\hline \multicolumn{4}{|l|}{ Amazonas } \\
\hline \multicolumn{4}{|l|}{ Ancash } \\
\hline \multicolumn{2}{|l|}{ Apurímac } & & $x$ \\
\hline Arequipa & $x$ & & \\
\hline Ayacucho & & $x$ & \\
\hline \multicolumn{4}{|l|}{ Cajamarca } \\
\hline \multicolumn{2}{|l|}{ Callao } & & $x$ \\
\hline \multicolumn{2}{|l|}{ Cusco } & & $x$ \\
\hline \multicolumn{4}{|l|}{ Huancavelica } \\
\hline \multicolumn{2}{|l|}{ Huánuco } & & $x$ \\
\hline Ica & $x$ & $x$ & \\
\hline \multicolumn{4}{|l|}{ Junín } \\
\hline La Libertad & $x$ & & \\
\hline Lambayeque & $x$ & $x$ & \\
\hline \multicolumn{4}{|l|}{ Lima } \\
\hline \multicolumn{2}{|l|}{ Loreto } & & $x$ \\
\hline \multicolumn{4}{|l|}{ Madre de Dios } \\
\hline \multicolumn{4}{|l|}{ Moquegua } \\
\hline \multicolumn{4}{|l|}{ Pasco } \\
\hline \multicolumn{4}{|l|}{ Piura } \\
\hline \multicolumn{2}{|l|}{ Puno } & $x$ & \\
\hline \multicolumn{4}{|l|}{ San Martín } \\
\hline \multicolumn{4}{|l|}{ Tacna } \\
\hline \multicolumn{4}{|l|}{ Tumbes } \\
\hline \multicolumn{2}{|l|}{ Ucayali } & & $x$ \\
\hline Desabastecimiento & $16 \%$ & $16 \%$ & $24 \%$ \\
\hline
\end{tabular}

Tabla 3. Desabastecimiento de medicamentos antihipertensivos según región política.

\begin{tabular}{|c|c|c|c|c|c|c|c|c|c|c|c|c|c|}
\hline Región & $\begin{array}{l}\text { Atenolol } 50 \\
\text { mg tab }\end{array}$ & $\begin{array}{l}\text { Atenolol } \\
100 \mathrm{mg} \\
\text { tab }\end{array}$ & $\begin{array}{l}\text { Carvedilol } \\
25 \mathrm{mg} \text { tab }\end{array}$ & $\begin{array}{c}\text { Carvedilol } \\
12.5 \mathrm{mg} \text { tab }\end{array}$ & $\begin{array}{l}\text { Carvedilol } \\
6.25 \mathrm{mg} \text { tab }\end{array}$ & $\begin{array}{l}\text { Captopril } \\
25 \mathrm{mg} \text { tab }\end{array}$ & $\begin{array}{c}\text { Enalapril } \\
\text { maleato } \\
20 \mathrm{mg} \text { tab }\end{array}$ & $\begin{array}{l}\text { Labetalol } 5 \\
\mathrm{mg} / \mathrm{ml} \text { iny }\end{array}$ & $\begin{array}{l}\text { Nitroprusiato } \\
\text { sódico } 50 \mathrm{mg} \\
\text { iny }\end{array}$ & $\begin{array}{c}\text { Amlodipino } \\
10 \mathrm{mg} \text { tab }\end{array}$ & $\begin{array}{l}\text { Amlodipino } \\
5 \mathrm{mg} \text { tab }\end{array}$ & $\begin{array}{l}\text { Bisoprolol } \\
\text { fumarato } 5 \\
\text { mg tab }\end{array}$ & $\begin{array}{l}\text { Nifedipino } \\
30 \mathrm{mg} \text { tab }\end{array}$ \\
\hline Amazonas & $x$ & & $x$ & $x$ & $x$ & & & $x$ & $x$ & & & $x$ & \\
\hline Ancash & $x$ & & & & $x$ & & & $x$ & $x$ & & & & \\
\hline Apurímac & $x$ & & $x$ & $x$ & $x$ & & & $x$ & $x$ & & & $x$ & \\
\hline Ayacucho & $x$ & $x$ & $x$ & $x$ & $x$ & $x$ & & $x$ & $x$ & $x$ & $x$ & $x$ & \\
\hline Cajamarca & $\mathrm{x}$ & & $x$ & & $x$ & & & $x$ & $x$ & & & $x$ & $x$ \\
\hline Callao & $\mathrm{x}$ & & & $x$ & $x$ & $x$ & & $x$ & $x$ & & & & \\
\hline Cusco & $x$ & & $x$ & $x$ & $x$ & & & $x$ & $x$ & & & $x$ & $x$ \\
\hline Huancavelica & $\mathrm{x}$ & $x$ & $\mathrm{x}$ & $x$ & $x$ & & & $x$ & $x$ & & & $x$ & \\
\hline Huánuco & $\mathrm{x}$ & & $x$ & $x$ & $x$ & & & $x$ & $x$ & & & $x$ & $x$ \\
\hline Ica & $\mathrm{x}$ & & $\mathrm{x}$ & $x$ & $x$ & & & $\mathrm{x}$ & $\mathrm{x}$ & & & $\mathrm{x}$ & $x$ \\
\hline Junín & $\mathrm{x}$ & & $x$ & $x$ & $x$ & & & $x$ & $x$ & & & $x$ & \\
\hline Lambayeque & $\mathrm{x}$ & $x$ & $\mathrm{x}$ & & $x$ & & & $x$ & $x$ & & & $x$ & \\
\hline Lima & $x$ & & & & & & & & & & & & \\
\hline Loreto & & & $x$ & $x$ & $x$ & & & $x$ & & & & & \\
\hline Madre de Dios & $x$ & & $x$ & & $x$ & & & $x$ & $x$ & $x$ & & $x$ & $x$ \\
\hline Moquegua & $x$ & & $x$ & $x$ & $x$ & & & $x$ & $x$ & $x$ & & $x$ & \\
\hline Pasco & $\mathrm{x}$ & $x$ & $\mathrm{x}$ & $x$ & $x$ & & & $\mathrm{x}$ & $x$ & $x$ & $x$ & $\mathrm{x}$ & $x$ \\
\hline Piura & $x$ & & $x$ & $x$ & $x$ & & & $x$ & $x$ & & & $x$ & \\
\hline Puno & $\mathrm{x}$ & $x$ & $x$ & $x$ & $x$ & & & $x$ & $x$ & & & $x$ & $x$ \\
\hline San Martín & $x$ & & & $x$ & $x$ & & & $x$ & $x$ & & & $x$ & \\
\hline Tacna & $\mathrm{x}$ & & & & & & & $x$ & & & & & \\
\hline Tumbes & $x$ & & & $x$ & $x$ & & & $x$ & $x$ & & & & \\
\hline Ucayali & $x$ & $x$ & $x$ & $x$ & $x$ & & & $x$ & $x$ & & $x$ & $x$ & \\
\hline Desabastecimi & ient $\Theta 6 \%$ & $24 \%$ & $76 \%$ & $76 \%$ & $92 \%$ & $8 \%$ & $4 \%$ & $96 \%$ & $88 \%$ & $20 \%$ & $12 \%$ & $76 \%$ & $32 \%$ \\
\hline
\end{tabular}


Revista del Cuerpo Médico del HNAAA, Vol. 14 Supl. №1 ( 2021 ) | SARS-CoV-2, COVID-19 y Pandemia

Percy Herrera-Añazco, Germán Valenzuela-Rodríguez, Luciana Torres-Pesantes, Carlos J. Toro-Huamanchumo

Tabla 4. Disponibilidad relativa de los antidiabéticos según región natural y tipo de establecimiento de salud.

\begin{tabular}{|c|c|c|c|c|c|c|c|c|c|c|}
\hline & \multicolumn{4}{|c|}{ Costa } & \multicolumn{3}{|c|}{ Sierra* } & \multicolumn{3}{|c|}{ Selva* } \\
\hline & CS (\%) & PS (\%) & $\mathrm{H}(\%)$ & IE $(\%)$ & CS $(\%)$ & PS (\%) & $\mathrm{H}(\%)$ & CS (\%) & PS (\%) & H (\%) \\
\hline Glibenclamida 5 mg & 98,1 & 95,2 & 100 & 100 & 94,5 & 96,5 & 81,8 & 93,3 & 95,3 & 100 \\
\hline Insulina isófona humana & 83,8 & 100 & 100 & 100 & 95,2 & 80 & 100 & 95 & 100 & 100 \\
\hline Metformina $850 \mathrm{mg}$ & 98,3 & 97,7 & 100 & 100 & 98 & 97,9 & 100 & 93,7 & 96 & 100 \\
\hline Metformina $500 \mathrm{mg}$ & 98,7 & 96,9 & 100 & 100 & 98,8 & 95,5 & 100 & 100 & 96,3 & 100 \\
\hline
\end{tabular}

*No hay institutos especializados en la sierra ni en la selva

CS: Centro de salud, PS: Puesto de salud, H: Hospital, IE: Instituto especializado

Tabla 5. Disponibilidad relativa de antihipertensivos según región natural y tipo de establecimiento.

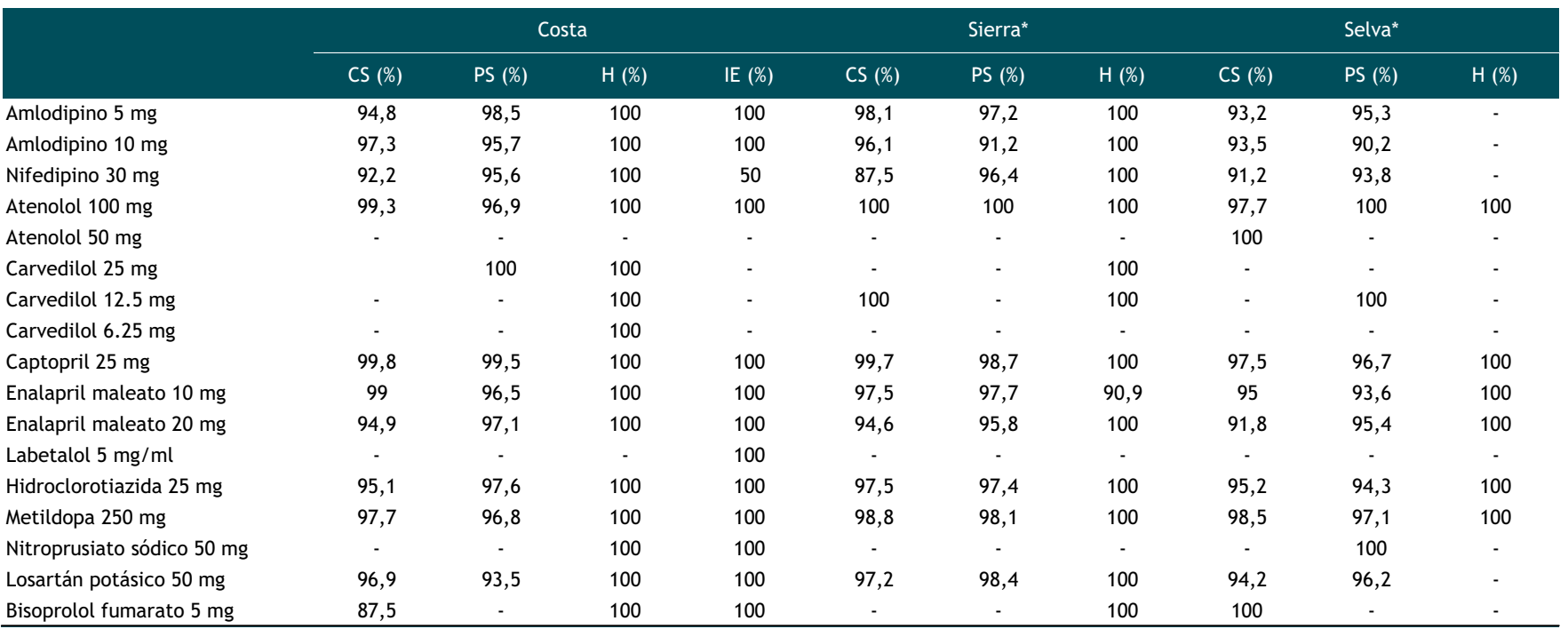

*No hay institutos especializados en la sierra ni en la selva

CS: Centro de salud, PS: Puesto de salud, H: Hospital, IE: Instituto especializado

Al evaluar la disponibilidad de los antidiabéticos, tres de ellos no se encontraban en ningún establecimiento de salud en por lo menos una región, siendo el medicamento más desabastecido la Metformina de $500 \mathrm{mg}$ en el $24 \%$ de los departamentos. (Tabla 2).

En el caso de los antihipertensivos, el Labetalol $5 \mathrm{mg} / \mathrm{ml}$ iny, Atenolol $50 \mathrm{mg}$ tab, Carvedilol $6,25 \mathrm{mg}$ tab estuvieron totalmente desabastecidos en más del $90 \%$ de los departamentos. En el caso del Labetalol, solo se encontró disponible en Lima en al menos un establecimiento; escenario similar al del Atenolol, en Loreto (Tabla 3).

Las tablas 3 y 4 muestran la disponibilidad relativa (\%) de los medicamentos según región natural y medicamento. En el caso de los antidiabéticos, solo los hospitales e institutos especializados de la costa tuvieron una disponibilidad del $100 \%$. Lo mismo sucedió en la selva, aunque solo en hospitales puesto que esta región no cuenta con institutos especializados. La insulina humana no se encontró en ningún puesto de salud de la sierra. Por otro lado, en el caso de los antihipertensivos, encontramos que varios medicamentos no se reportaron disponibles en diferentes establecimientos de las regiones. Atenolol $50 \mathrm{mg}$ solo se encontró en centros de salud de la selva, mientras que el carvedilol $6,25 \mathrm{mg}$ solo en hospitales de la costa.

\section{DISCUSIÓN}

El desabastecimiento de medicamentos es un problema global reconocido por la Organización Mundial de la Salud. Según una encuesta realizada en 2014 a farmacéuticos de hospitales de 36 países europeos, el 66\% experimentó este problema a diario o semanalmente ${ }^{(17)}$. En el caso de medicamentos esenciales para enfermedades no comunicables, un estudio a nivel mundial mostró que la disponibilidad de medicamentos (de marca de origen o genéricos) en el sector público en países de ingresos bajos fue de $43,3 \%$, y de $57,6 \%$ en los países de ingresos medios ${ }^{(18)}$.

En nuestro país, el desabastecimiento de medicamentos en las farmacias de los establecimientos de salud también es un problema mostrado en algunas investigaciones. Un estudio que utilizó la Encuesta Nacional de Satisfacción de Usuarios del Aseguramiento Universal en Salud (ENSUSALUD) del 2014 encontró que el $30,6 \%$ de pacientes que acuden a las farmacias de distintas instituciones prestadoras de servicios de salud tuvo una dispensación incompleta o no recibió ningún medicamento, siendo mayor si las farmacias pertenecían a establecimientos del MINSA ${ }^{(14)}$. De igual forma, en el caso de medicamentos para enfermedades específicas, un estudio que, al igual que el nuestro utilizó el SISMED, encontró que los antidepresivos y antipsicóticos se 
encontraron disponibles en alrededor de dos tercios de los hospitales, y en menos del $20 \%$ de centros y puestos de salud del MINSA ${ }^{(15)}$.

Específicamente para antidiabéticos y antihipertensivos, el Prospective Urban Rural Epidemiological study, que incluye 626 comunidades de 20 países, mostró que la metformina estuvo disponible en el $86,1 \%$ de farmacias en países de ingresos medios bajos y $64,7 \%$ de farmacias en países de ingresos bajos $^{(19)}$. En el caso de insulina, ésta estuvo disponible en el $29,3 \%$ de farmacias en países de ingresos medio bajo y de 10,3\% de farmacias en países de ingresos bajos $^{(19)}$. Para el caso de los antihipertensivos, la disponibilidad de dos o más clases de antihipertensivos fue menor en países de ingresos medios y bajos que en países de ingresos altos ${ }^{(20)}$. De igual forma, la proporción de comunidades con cuatro clases de antihipertensivos disponibles fue de $71 \%$ en países de ingresos medios altos, $47 \%$ en países de ingresos medios bajos y $13 \%$ en países de ingresos bajos ${ }^{(20)}$.

Bajo la premisa mencionada anteriormente, nuestros hallazgos de desabastecimiento de antihipertensivos y antidiabéticos, siendo mayor en el caso de estos últimos, no son sorprendentes. Específicamente para el caso de antihipertensivos, un estudio usando la ENSUSALUD del 2016 encontró que el acceso de estos medicamentos fue mayor en las farmacias del Seguro Social (EsSalud) y de clínicas privadas que en las farmacias del MINSA. De igual forma, fue menor en los hospitales que en centros de menor nivel de complejidad. No obstante, este estudio no cuantificó el tipo de antihipertensivos ni hizo una evaluación a nivel regional como el nuestro ${ }^{(21)}$.

El desabastecimiento de ambos grupos de medicamentos tiene varias aristas que incluyen la imposibilidad en algunos casos de remplazarlos por medicamentos de la misma clase cuando sea posible ${ }^{(22)}$. En el 2015 en España, en 95 de 160 medicamentos desabastecimientos, existían como alternativa otros medicamentos con el mismo principio activo y para la misma vía de administración ${ }^{(22)}$. La ausencia de medicamentos similares con que reemplazarlos puede aumentar la posibilidad de eventos adversos incluso fatales ${ }^{(23)}$. En EEUU se han registrado casos de muerte debido al uso de alternativas inadecuadas o a errores de medicación relacionados con los medicamentos alternativos ${ }^{(23)}$. De igual forma, el desabastecimiento desvía a los médicos de su actividad asistencial y, en ocasiones, les obliga a emplear alternativas menos adecuadas o con menor experiencia de uso $^{(22)}$. Por otro lado, implican un incremento de los costes para el sistema de salud y para el paciente. En Perú, por ejemplo, la poca disponibilidad de antidiabéticos el sector público resulta en la compra de Insulina hasta de 3 veces de su precio y Glibenclamida en 15 veces, en farmacias del sector privado ${ }^{(24)}$

En nuestro país, los antidiabéticos y antihipertensivos, forman parte de los medicamentos "no estratégicos", y aunque el MINSA, como ente rector tiene la posibilidad de hacer compras corporativas, solo un cuarto de las compras nacionales de medicamentos se hace por medio de esta vía, dejando la compra del resto a la gestión de los gobiernos regionales caracterizados por la ineficiencia en la cadena de procesos del abastecimiento ${ }^{(25)}$. Aunque es posible hacer requerimientos complementarios para resolver los problemas de abastecimiento, las negociaciones son lentas y engorrosas. Como consecuencia, se recurren a compras urgentes y usualmente costosas ${ }^{(21,25)}$.

La crisis sanitaria por el COVID-19 desnudó aún más la gravedad de esta situación, siendo necesario crear una entidad independiente que centralice las gestiones, A pesar de ello, los problemas de gestión de los gobiernos regionales limitaron la efectividad de estas medidas. A pesar de que no podemos afirmar que lo mostrado en nuestro estudio sea peor que en épocas "pre crisis", por lo visto en el abastecimiento de otros insumos médicos tales como el oxígeno, es posible que sea $a s^{\prime(26)}$. Sin un adecuado control de la HTA y la DM es posible que parte de las muertes durante la etapa de la pandemia estudiada y a mediano plazo se deban a complicaciones asociadas al mal control de estas enfermedades ${ }^{(12)}$

La HTA y la DM, sobre todo descompensadas, están asociadas a una mayor severidad del COVID-19 durante la admisión hospitalaria y con mayor riesgo de muerte ${ }^{(27,28)}$. Por ello, se recomienda mantener la terapia antihipertensiva en todos los casos y especialmente si el paciente usara inhibidores del sistema renina angiotensina, en el contexto de la infección por COVID-19 ${ }^{(29)}$. Para el caso de los diabéticos con COVID-19, la terapia debe ser individualizada por el riesgo de descompensación inducida por las terapias que el paciente recibe ${ }^{(30)}$. Por ello, la disponibilidad y acceso a varias opciones terapéuticas es crucial.

Se han planteado algunas estrategias para mejorar el abastecimiento de estos medicamentos. En primer lugar, promover la prescripción y provisión de medicamentos genéricos en todas las entidades públicas e incrementar la oferta de medicamentos en establecimientos públicos ${ }^{(25)}$. De igual forma, se debe garantizar el financiamiento anual de medicamentos para el sector público, mejorando la gestión de programación, compra, distribución y disposición de medicamentos y evitar retrasos en las compras corporativas del Centro Nacional de Abastecimiento de Recursos Estratégico en Salud ${ }^{(25)}$.

Nuestro estudio tiene limitaciones. En primer lugar, recogemos la información oficial del SISMED, y es posible que no haya recogido información de la totalidad de establecimientos de salud del país. En segundo lugar, hemos evaluado los antidiabéticos y antihipertensivos que figura en el petitorio nacional, que no necesariamente incluye todos los medicamentos existentes para el manejo de la HTA o la DM. En tercer lugar, se ha considerado la definición de desabastecimiento de la SISMED que significa cero tabletas o pastillas, existiendo la posibilidad de que, aunque haya una pastilla o tableta, el sistema lo cataloga como no desabastecido, lo que sobreestimaría la capacidad de los establecimientos de salud para dar un tratamiento completo. En cuarto lugar, se evaluó el desabastecimiento en la primera etapa de la la pandemia y existe la posibilidad de que haya mejorado posteriormente. Por último, al no poder comparar el abastecimiento actual con lo que había antes de la pandemia, no podemos afirmar si este ha disminuido.

En conclusión, existe un porcentaje importante de desabastecimiento de antihipertensivos y antidiabéticos en 
los establecimientos del Ministerio de Salud, lo que podría exponer a los pacientes a un riesgo mayor de complicaciones y mortalidad.

\section{REFERENCIAS BIBLIOGRÁFICAS}

1. Olsen MH, Angell SY, Asma S, et al. A call to action and a life course strategy to address the global burden of raised blood pressure on current and future generations: the Lancet Commission on hypertension. Lancet. 2016;388(10060):2665-2712. doi:10.1016/S0140-6736(16)31134-5.

2. Tao Z, Shi A, Zhao J. Epidemiological Perspectives of Diabetes. Cell Biochem Biophys. 2015;73(1):181-185. doi:10.1007/s12013-0150598-4

3. Arredondo A, Azar A, Recamán AL. Diabetes, a global public health challenge with a high epidemiological and economic burden on health systems in Latin America. Glob Public Health. 2018;13(7):780-787. doi:10.1080/17441692.2017.1316414

4. Herrera-Añazco P, Amaya E, Atamari-Anahui N, Ccorahua-Rios M, Hernandez AV. Association between social determinants of health and trends in prevalence of hypertension in patients of the Peruvian Ministry of Health. Trop Med Int Health. 2019;24(12):1434-1441. doi:10.1111/tmi.13318

5. Villarreal-Zegarra D, Carrillo-Larco RM, Bernabe-Ortiz A. Short-term trends in the prevalence, awareness, treatment, and control of arterial hypertension in Peru [published online ahead of print, 2020 Jun 9]. J Hum Hypertens. 2020;10.1038/s41371-020-0361-1. doi:10.1038/s41371-020-0361-1

6. Villena JE. Diabetes Mellitus in Peru. Ann Glob Health. 2015;81(6):765-775. doi:10.1016/j.aogh.2015.12.018

7. Ccorahua-Ríos MS, Atamari-Anahui N, Miranda-Abarca I, CamperoEspinoza AB, Rondón-Abuhadba EA, Pereira-Victorio CJ. Type 2 diabetes mellitus prevalence between 2005 and 2018 in population under 30 using data from the Ministry of Health of Peru. Prevalencia de la diabetes mellitus tipo 2 en población menor de 30 años para el período de 2005 a 2018 con datos del Ministerio de Salud de Perú. Medwave. 2019;19(10):e7723. doi:10.5867/medwave.2019.10.7723.

8. Miranda JJ, Herrera VM, Chirinos JA, et al. Major cardiovascular risk factors in Latin America: a comparison with the United States. The Latin American Consortium of Studies in Obesity (LASO). PLoS One. 2013;8(1):e54056. doi:10.1371/journal.pone.0054056

9. Herrera-Añazco P, Adrián V. Hernández, Mezones-Holguin E. Diabetes mellitus y nefropatía diabética en el Perú. Rev Nefrol Dial Traspl. [Internet]. 2017 [citado 19 de septiembre de 2021];35(4):229-37. D i s p o n i b l e e $n$ : https://www.revistarenal.org.ar/index.php/rndt/article/view/46

10. Soto A. Barreras para una atención eficaz en los hospitales de referencia del Ministerio de Salud del Perú: atendiendo pacientes en el siglo XXI con recursos del siglo XX. Rev Peru Med Exp Salud Publica. 2019;36(2):304-311. doi:10.17843/rpmesp.2019.362.4425

11. Huamaní C, TimanáRuiz R, Pinedo J, Pérez J, Vásquez L. Condiciones estimadas para controlar la pandemia de COVID-19 en escenarios de pre y poscuarentena en Perú. Rev Peru Med Exp Salud Publica. 2020;37(2):195-202. doi: 10.17843/rpmesp.2020.372.5405

12. FT Visual \& Data Journalism team. 2020. Coronavirus tracked: the latest figures as countries start to reopen | Free to read. Financial Time [internet].2020 [citado 19 de septiembre de 2021]. Disponible en: https: / / www.ft.com/content/a26fbf7e-48f8-11ea-aeb3$955839 \mathrm{e} 06441$

13. Sala Situacional COVID-19 Perú. [internet]. MINSA.Peru.2020 [citado 24 de julio de 2021 ]. D is pon ible en: https://covid19.minsa.gob.pe/sala situacional.asp

14. Mezones-Holguin E, Solis-Cóndor R, Benites-Zapata VA, GarnicaPinazo Holguín G, Marquez-Bobadilla E, et al. Diferencias institucionales en el insuficiente acceso efectivo a medicamentos prescritos en instituciones prestadoras de servicios de Salud en Perú: análisis de la Encuesta nacional de Satisfacción de Usuarios de los Servicios de Salud (Ensusalud 2014). Rev Peru Med Exp Salud Publica.
2016;33(2):205-14. doi: 10.17843/rpmesp.2016.332.2197

15. Hodgkin D, Piazza M, Crisante M, Gallo C, Fiestas F. Disponibilidad de medicamentos psicotrópicos en establecimientos del Ministerio de Salud del Perú, 2011. Rev Peru Med Exp Salud Publica. [Internet] 2014 [Citado el 18 de setiembre del 2021];31(4):660-668. Disponible en: http: / /www.scielo.org.pe/scielo.php?script=sci_arttext\&pid=S1726 $-46342014000400007$

16. World Health Organization Centre for Drug Statistics Methodology [Internet]. Structure and principles WHOCC. Oslo. 2018. [Citado el 24

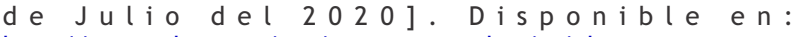
http://www.whocc.no/atc/structure_and_principles

17. European Association of Hospital Pharmacists. Medicine shortages in European Hospitals. Results of the largest pan- European survey on medicines supply shortages in the hospital sector, its prevalence, nature and impacts for patient care [Internet]. EAHP. Brucelas. 2014. [Citado el 24 de julio del 2020]. Disponible en: http://www.eahp.eu/practiceand-policy/medicines-shortages

18. Ewen M, Zweekhorst M, Regeer B, Laing R. Baseline assessment of WHO's target for both availability and affordability of essential medicines to treat non-communicable diseases. PLoS One. 2017;12(2):e0171284. doi:10.1371/journal.pone.0171284

19. Chow CK, Ramasundarahettige C, Hu W, et al. Availability and affordability of essential medicines for diabetes across high-income, middle-income, and low-income countries: a prospective epidemiological study. Lancet Diabetes Endocrinol. 2018;6(10):798808. doi:10.1016/S2213-8587(18)30233-X

20. Attaei MW, Khatib R, McKee M, et al. Availability and affordability of blood pressure-lowering medicines and the effect on blood pressure control in high-income, middle-income, and low-income countries: an analysis of the PURE study data. Lancet Public Health. 2017;2(9):e411-e419. doi:10.1016/S2468-2667(17)30141-X

21. Tenorio Mucha JM. Acceso a medicamentos para controlar la hipertensión arterial prescritos en consulta externa en las Instituciones Prestadoras de Servicios de Salud (IPRESS) en Perú, ENSUSALUD 2016. Perú: Maestro en Ciencias en Investigación Epidemiológica, Tesis de maestría, Universidad Peruana Cayetano Heredia. [Internet] 2019. [Citado el 24 de julio del 2020]. Disponible en: http://repositorio.upch.edu.pe/handle/upch/6611

22. Eusko Jaurlaritzaren Argitalpen Z erbitzu Nagusia.Desabastecimientos de medicamentos: un problema sin resolver [Internet].. INFAC. Pais Vasco. 2015. [Citado el 24 de julio del 2020]. Disponible en: https://bit.ly/3lDmwi3

23. Fox ER, Sweet BV, Jensen V. Drug shortages: a complex health care crisis. Mayo Clin Proc. 2014;89(3):361-373. doi:10.1016/j.mayocp.2013.11.014

24. Cardenas MK, Miranda JJ, Beran D. Delivery of type 2 diabetes care in low- and middle-income countries: lessons from Lima, Peru. Diabet Med. 2016;33(6):752-60. doi: 10.1111/dme.13099.

25. Ugarte 0 . Estrategias para mejorar el acceso a medicamentos en el Perú. An Fac med. 2019;80(1):104-8. doi: 10.15381/anales.v80i1.15878

26. Millán Valencia A. Coronavirus en Perú | La escasez de oxígeno por el covid-19 que puso en alerta al gobierno. [Internet] UK. BBC News. 2020. [Citado en 29 de julio del 2020]. Disponible en: https://www.bbc.com/mundo/noticias-america-latina-53025355

27. Hu Y, Sun J, Dai Z, Deng H, Li X, Huang Q, et al. Prevalence and severity of coronavirus disease 2019 . A systematic review and metaanalysis. J Clin Virol 2020;137:104731. doi: 10.1016/j.jcv.2020.104371.

28. Li X, Xu S, Yu M, Wang K, Tao Y, Zhou Y et al. Risk Factors for severity and mortality in adult COVID-19 in patients in Wuhan. J Allergy Clin Immunol 2020;146(1):110-118. doi: 10.1016/j.jaci.2020.04.006

29. Tanriverdi O, Sengul H. The effect of Coronavirus Disease 2019 on Cardiovascular Diseases. Arq. Bras. Cardiol 2020;114(5). doi: $10.36660 /$ abc. 20200273

30. Kumar Singh A, Gupta R, Ghosh A, Misra A. Diabetes in COVID-19. Prevalence, pathophysiology, prognosis and practical considerations. Diabetes Metab Syndr 2020;14(4):303-310. doi: 10.1016/j.dsx.2020.04.004. 